Agricultural Journal 7 (4): 255-259, 2012

ISSN: $1816-9155$

(C) Medwell Journals, 2012

\title{
Response Surface Methodology (RSM) for Optimization of the Microwave-Assisted Extraction of Polysaccharides from Catathelasma ventricosum
}

\author{
${ }^{1,2}$ Ling Zhao, ${ }^{1,2}$ Kaiyu Wang, ${ }^{1}$ Dexiang Wang, ${ }^{1}$ Chang Liu and ${ }^{1,2}$ Wei Zhang \\ ${ }^{1}$ College of Verterinary Medicine, Sichuan Agricultural University, Ya' an, China \\ ${ }^{2}$ Key Laboratory of Animal Disease and Human Health of Sichuan Province, \\ Sichuan Agricultural University, Ya'an, China
}

\begin{abstract}
Microwave-assisted extraction was investigated for extraction of polysaccharides from Catathelasma ventricosum using Response Surface Methodology (RSM). The effects of operating conditions (extraction time, microwave power and ratio of water to raw material) on the extraction yield of polysaccharides were studied. A Box-Behnken Design was used for experimental design and analysis of the results to obtain the optimal processing parameters. Among the three extraction parameters, the microwave power was the most significant factor to affect the yield of polysaccharides from Catathelasma ventricosum. In addition, microwave power and extraction time had significant interaction effects. A mathematical model with high determination coefficient was gained and could be employed to optimize polysaccharides extraction and the optimal conditions were extraction time $5 \mathrm{~min}$, microwave power $420 \mathrm{~W}$, ratio of water to raw material 25:1. Under these conditions, the experimental yield of polysaccharide was $7.83 \pm 0.19 \%$ which was close to the predicted value.
\end{abstract}

Key words: Response surface analysis, Catathelasma ventricosum, polysaccharides, optimization, microwaveassisted extraction, China

\section{INTRODUCTION}

Hot water extraction is the most conventional extraction method for polysaccharides. It should be noted that traditional hot water extraction of polysaccharides is time and solvent consuming and has low efficiency (Ye and Jiang, 2011; Liu et al., 2009; Sun et al., 2010). Moreover, thermal extraction over a long time can cause the degradation of polysaccharides. Recently, alternative extraction techniques such as ultrasonic, microwave assisted extraction and enzyme-assisted extraction with shorter time or enhanced yields had been also reported (Chen et al., 2010; Zhang et al., 2011; Pan et al., 2010). Many reports have been published on the extraction of polysaccharides from plants using microwave-assisted extraction (Bagherian et al., 2011; Wang et al., 2009, 2010). Microwave-assisted extraction was different other heating methods that solutions are heated so efficiently that reaction timescales are dramatically reduced. Microwave-assisted Extraction (MAE) utilizes the energy of microwaves to cause molecular movement and rotation of liquids with a permanent dipole, leading to rapid heating of the solvent and the sample. It has advantages over conventional extraction techniques such as improved efficiency, reduced extraction time, low solvent consumption
(Buldini et al., 2002). Wang et al. (2010) reported that MAE had great potential and efficiency, the extracting time was much shorter and the yield and of polysaccharides increased greatly. Catathelasma ventricosum is a wild and edible fungus distributed in Heilongjiang, Sichuan, Xizhang and Yunnan provinces of China which is a Tricholomataceae fungus belonging to the Basidiomycetes.

Numerous reports have revealed that polysaccharides isolated from mushrooms have broad spectrum of pharmacological properties such as antioxidant, antiinflammatory, anticancer and immunostimulatory activity (Bin, 2010; Diniz et al., 2003; Sun et al., 2008).

And in the other study, researchers had found antioxidant activity in vitro and to have effect on differentiation of preadipocyte. However, no information has yet been found on the application of MAE for the extraction of Catathelasma ventricosum. The objective of this study was to optimize extraction condition of the polysaccharides from the fruiting body of Catathelasma ventricosum using MAE.

\section{MATERIALS AND METHODS}

The fruiting bodies of Catathelasma ventricosum were purchased from Chuxiong, Yunnan province, China.

Corresponding Author: Kaiyu Wang, Key Laboratory of Animal Disease and Human Health of Sichuan Province, Sichuan Agricultural University, Ya'an, China 
Phenol was form BeiJing Solarbio S\&T Co., Ltd. (Beijing, China), sulphuric acid, ethanol and glucose were from the Cheng Du Kelong Chemical Factory (Chengdu, China). All other chemicals were of analytical grade.

Extraction of crude polysaccharides from Catathelasma ventricosum: Microwave-assisted extraction was carried out using an ordinary household oven (Galanz, WD700G, China) with adjustable power settings ranging from 100-700 W. The fruiting bodies of Catathelasma ventricosum was soaked in 4 volumes of $95 \%$ ethanol twice for $24 \mathrm{~h}$ to remove some colored materials, oligosaccharides and some small molecule materials. After removing supernatant, precipitation was dried at $60^{\circ} \mathrm{C}$. The pretreated powder $(10.0 \mathrm{~g})$ was added a certain volume of distilled water and soaked for $30 \mathrm{~min}$ then transferred into the chamber of the microwave oven. The pretreated Catathelasma ventricosum powder was extracted for 3 times in a designed extraction time, microwave power and ratio of water to raw material and then mixed the three extract liquids together. The supernatant was concentrated in a rotary evaporator under reduced pressure at $60^{\circ} \mathrm{C}$. The resulting solution was precipitated by the addition of $80 \%(\mathrm{v} / \mathrm{v})$ ethanol and the precipitate was collected by sucking filtration and washed with $100 \%$ cold ethanol, acetone and finally dried at $55^{\circ} \mathrm{C}$ to obtain crude polysaccharide of Catathelasma ventricosum (MCP). The total sugar content of polysaccharide was measured by the phenol-sulfuric acid method (Dubois et al., 1956), glucose was used as standard and the results were then expressed as glucose equivalents.

Experimental design of RSM: RSM was used to estimate the influence of three independent variables and the optimal extraction condition of crude polysaccharides from Catathelasma ventricosum. Three independent variables $\left(\mathrm{X}_{1}\right.$, extraction time; $\mathrm{X}_{2}$, microwave power, $\mathrm{X}_{3}$, ratio of water to raw material) at three different levels were employed. Box-Behnken Design (BBD) was used in this optimization study. The range of independent variables and their levels were shown in Table 1. Extraction yield (y) was taken as the response for the combination of the independent variables shown in Table 2. Experimental runs were randomized to minimize the effects of unexpected variability in the observed response. Experiment data were fitted to a second-order polynomial model and regression coefficients obtained which was as:

$$
\mathrm{y}=\mathrm{A}_{0}+\sum_{\mathrm{i}=1}^{3} \mathrm{~A}_{\mathrm{i}} \mathrm{X}_{\mathrm{i}}+\sum_{\mathrm{i}=1}^{3} \mathrm{~A}_{\mathrm{iij}} \mathrm{X}_{\mathrm{i}}^{2}+\sum_{\mathrm{i}=1}^{2} \sum_{\mathrm{j}=\mathrm{i}+1}^{3} \mathrm{~A}_{\mathrm{ij}} \mathrm{X}_{\mathrm{ij}}
$$

Where:

$\mathrm{y}=$ Represent the response function

$\mathrm{A}_{0}=$ An intercept
Table 1: Indepedent variables and their levels used in the response surface design

\begin{tabular}{lrrr}
\hline & Levels & & \\
& -1 & 0 & 1 \\
\hline Factors & \multicolumn{1}{c}{-1} & 4 & 5 \\
$\mathrm{X}_{1}$ extraction time/min & 3 & 560 & 700 \\
$\mathrm{X}_{2}$ microwave power $/ \mathrm{w}$ & 420 & 20 & 25 \\
$\mathrm{X}_{3}$ ratio of water to raw material & 15 & & \\
\hline
\end{tabular}

Table 2: Box-Behnken experimental design and results for extraction yield

\begin{tabular}{lcccc}
\hline Run & $\begin{array}{c}\mathrm{X}_{1} / \text { extraction } \\
\text { time (min) }\end{array}$ & $\begin{array}{c}\mathrm{X}_{2} / \text { microwave } \\
\text { power }(\mathrm{W})\end{array}$ & $\begin{array}{c}\mathrm{X}_{3} / \text { ratio of water } \\
\text { to raw material }\end{array}$ & Yield (\%) \\
\hline 1 & $0 / 4$ & $0 / 560$ & $0 / 20$ & 7.01 \\
2 & $1 / 5$ & $1 / 700$ & $0 / 20$ & 5.64 \\
3 & $-1 / 3$ & $1 / 700$ & $0 / 20$ & 6.67 \\
4 & $-1 / 3$ & $-1 / 420$ & $0 / 20$ & 3.42 \\
5 & $0 / 4$ & $1 / 700$ & $1 / 25$ & 7.47 \\
6 & $1 / 5$ & $0 / 560$ & $1 / 25$ & 6.87 \\
7 & $1 / 5$ & $-1 / 420$ & $0 / 20$ & 7.21 \\
8 & $0 / 4$ & $1 / 700$ & $-1 / 15$ & 7.29 \\
9 & $0 / 4$ & $0 / 560$ & $0 / 20$ & 6.71 \\
10 & $0 / 4$ & $-1 / 420$ & $1 / 25$ & 7.24 \\
11 & $0 / 4$ & $0 / 560$ & $0 / 20$ & 7.24 \\
12 & $-1 / 3$ & $0 / 560$ & $-1 / 15$ & 6.65 \\
13 & $0 / 4$ & $0 / 560$ & $0 / 20$ & 7.27 \\
14 & $-1 / 3$ & $0 / 560$ & $1 / 25$ & 6.51 \\
15 & $1 / 5$ & $0 / 560$ & $-1 / 15$ & 5.78 \\
16 & $0 / 4$ & $-1 / 420$ & $-1 / 15$ & 5.74 \\
17 & $0 / 4$ & $0 / 560$ & $0 / 20$ & 6.50 \\
\hline
\end{tabular}

$\mathrm{A}_{\mathrm{i}}, \mathrm{A}_{\mathrm{ii}}$ and $\mathrm{A}_{\mathrm{ij}}=$ The coefficients of the linear, quadratic and interactive terms, respectively

$\mathrm{X}_{\mathrm{i}}$ and $\mathrm{X}_{\mathrm{ij}} \quad=$ Represent levels of the independent variables

The model evaluated the effect of each independent variable to a response. Three additional confirmation experiments were conducted to verify the validity of the statistical experimental strategies.

Statistical analysis: The Design Expert Software (Version 8.0.6, Stat-Ease. Inc., Minneapolis, MN) was used to analyze the experimental data. The significance of each coefficient was determined using the F-test and p-value. The $\mathrm{p}<0.05$ was considered to be statistically significant.

\section{RESULTS AND DISCUSSION}

Model fitting: The experimental data for extraction yield of the crude polysaccharides under different treatment conditions are shown in Table 2.

The statistical analysis indicated that the proposed regression model for yield was adequate, possessing to significant lack of fit and with satisfactory values of the $\mathrm{R}^{2}$ for all the responses. The $\mathrm{R}^{2}$ values were 0.88 for yield. The model can fit well with the actual data when approaches one. 
Analysis of response surface: Predicted response y for the yield of polysaccharides could be expressed by the following second-order polynomial equation in terms of coded values (Table 3 ).

$$
\begin{aligned}
\mathrm{y}= & 6.95+0.28 \mathrm{X}_{1}+0.43 \mathrm{X}_{2}+0.33 \mathrm{X}_{3}-1.21 \mathrm{X}_{1} \mathrm{X}_{2}+ \\
& 0.31 \mathrm{X}_{1} \mathrm{X}_{3}-0.33 \mathrm{X}_{2} \mathrm{X}_{3}-0.85 \mathrm{X}_{1}^{2}-0.37 \mathrm{X}_{2}^{2}+0.35 \mathrm{X}_{3}^{2}
\end{aligned}
$$

Where:

$$
\begin{aligned}
\mathrm{y}= & \text { The yield of } \mathrm{MCP} \\
\mathrm{X}_{1}, \mathrm{X}_{2} \text { and } \mathrm{X}_{3}= & \text { The coded values for extraction time, } \\
& \text { microwave power and ratio of water to } \\
& \text { raw material, respectively }
\end{aligned}
$$

The analysis of variance for these modes was given in Table 4. In Table 4 , the linear coefficients $\left(\mathrm{X}_{2}\right)$, the quadratic term coefficient $\left(\mathrm{X}_{1}, \mathrm{X}_{3}, \mathrm{X}_{2} \mathrm{X}_{3}, \mathrm{X}_{1} \mathrm{X}_{3}, \mathrm{X}_{2}^{2}, \mathrm{X}_{3}^{2}\right)$ and the interaction coefficient $\left(\mathrm{X}_{1} \mathrm{X}_{2}\right)$ were found significant $(\mathrm{p}<0.05)$. The other coefficients $\left(\mathrm{X}_{1}, \mathrm{X}_{3}, \mathrm{X}_{2} \mathrm{X}_{3}\right.$, $\left.\mathrm{X}_{1} \mathrm{X}_{3} \mathrm{X}_{2}^{2}, \mathrm{X}_{3}^{2}\right)$ were not significant $(\mathrm{p}>0.05)$. The full model

Table 3: Analysis of variance for the fitted quadratic polynomial model of extraction of polysaccharides

\begin{tabular}{lcclcl}
\hline Parameters & $\begin{array}{c}\text { Sum of } \\
\text { squares }\end{array}$ & $\begin{array}{c}\text { Degrees of } \\
\text { freedom }\end{array}$ & $\begin{array}{l}\text { Mean } \\
\text { square }\end{array}$ & F-value & p-value \\
\hline Model & 13.68 & 9 & 1.52 & 5.75 & 0.0155 \\
Residual & 1.85 & 7 & 0.26 & & \\
Lack of fit & 1.40 & 3 & 0.47 & 4.19 & 0.0999 \\
Pure error & 0.45 & 4 & 0.11 & & \\
Cor total & 15.53 & 16 & & & \\
\hline
\end{tabular}

Table 4: Regression coefficients estimate and their significance test for the Quadratic Polynomial Model

\begin{tabular}{lcccrr}
\hline Parameters & $\begin{array}{c}\text { Sum of } \\
\text { squares }\end{array}$ & $\begin{array}{c}\text { Degrees of } \\
\text { freedom }\end{array}$ & $\begin{array}{l}\text { Mean } \\
\text { square }\end{array}$ & F-value & p-value \\
\hline $\mathrm{X}_{1}$ & 0.63 & 1 & 0.63 & 2.40 & 0.1652 \\
$\mathrm{X}_{2}$ & 1.49 & 1 & 1.49 & 5.65 & 0.0492 \\
$\mathrm{X}_{3}$ & 0.86 & 1 & 0.86 & 3.25 & 0.1142 \\
$\mathrm{X}_{1} \mathrm{X}_{2}$ & 5.81 & 1 & 5.81 & 21.98 & 0.0022 \\
$\mathrm{X}_{1} \mathrm{X}_{3}$ & 0.38 & 1 & 0.38 & 1.43 & 0.2705 \\
$\mathrm{X}_{2} \mathrm{X}_{3}$ & 0.43 & 1 & 0.43 & 1.64 & 0.2417 \\
$\mathrm{X}_{1} \mathrm{X}_{1}$ & 3.01 & 1 & 3.01 & 11.40 & 0.0118 \\
$\mathrm{X}_{2} \mathrm{X}_{2}$ & 0.56 & 1 & 0.56 & 2.13 & 0.1880 \\
$\mathrm{X}_{3} \mathrm{X}_{3}$ & 0.53 & 1 & 0.53 & 1.99 & 0.2012 \\
\hline
\end{tabular}

filled, Eq. 2 was made 3-dimensional and contour plots to predict the relationships between the independent variables and the dependent variables.

Optimization of the process: The graphical representations of the regression (Eq. 2) called the response surfaces and the contour plots were obtained using Design-Expert 8.0. 6. As shown in Fig. 1-3, 3-dimensional response surface plots and 2-dimensional contour plots were useful to see interaction effects of the factors on the responses. They provide a method to visualize the relationship between responses and experimental levels of each variable and the type of interactions between two test variables.

Figure 1 shows the effects of extraction time and microwave power on the extraction yield of MCP at extraction time of zero level. It can be seen that the extraction time and microwave power had significant interaction effects on the yield of MCP. Polysaccharides yield was increased significantly when the microwave power was increased in the range from $420-700 \mathrm{~W}$ in relatively short extraction time. On the contrary, the yield of polysaccharides was decreased in the range from $420-700 \mathrm{~W}$ in longer extraction time. It indicated that the greater yield could be obtained when the moderate microwave power and extraction time were selected.

Figure 2 shows the effects of extraction time and ratio of water to raw material on the extraction yield of MCP at microwave power of zero level. The extraction yield of polysaccharides increased slightly with increasing of ratio of water to raw material from 15:1-25:1. And the yield of $\mathrm{MCP}$ increased with increasing of extraction time from $3 \mathrm{~min}$ to a threshold level, beyond this level, polysaccharides yield slightly decreased.

Figure 3 shows the effects of microwave power and ratio of water to raw material on the extraction yield of $\mathrm{MCP}$ at extraction time of zero level. The extraction yield of polysaccharides increased gradually with increasing of ratio of water to raw material from 15:1-25:1. The yield of

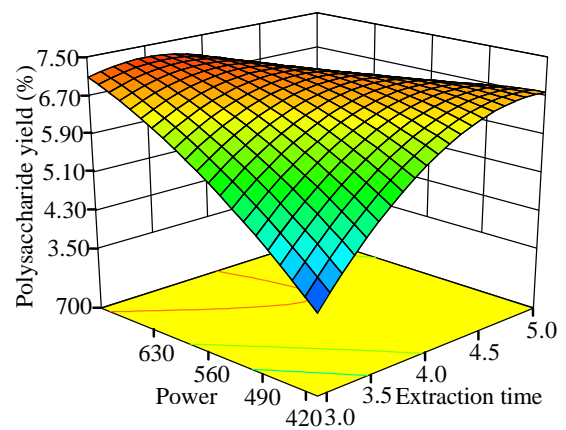

Design-Expert Software

Factor coding: Actual

Polysaccharide yield

7.47

3.42

$\mathrm{X}_{1}=\mathrm{A}$ : Extraction time

$\mathrm{X}_{2}=\mathrm{B}$ : Power

Actual factor

C: Ratio of water to raw material $=20$

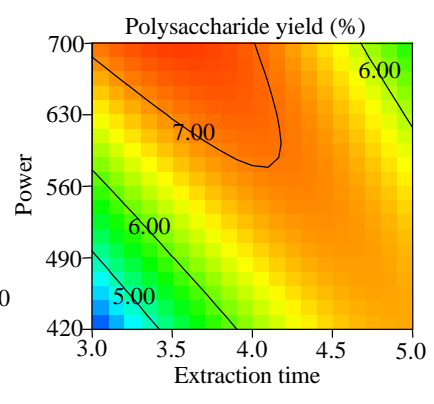

Fig. 1: Response surface plot and contour of microwave power and extraction time and their mutual interactions on the yield of polysaccharides 


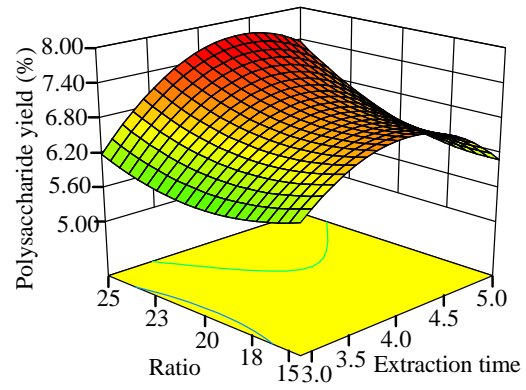

Design-Expert Software

Factor coding: Actual

Polysaccharide yield

7.47

3.42

$\mathrm{X}_{1}=\mathrm{A}$ : Extraction time

$\mathrm{X}_{2}=\mathrm{B}$ : Power

Actual factor

C: Ratio of water to raw material $=20$

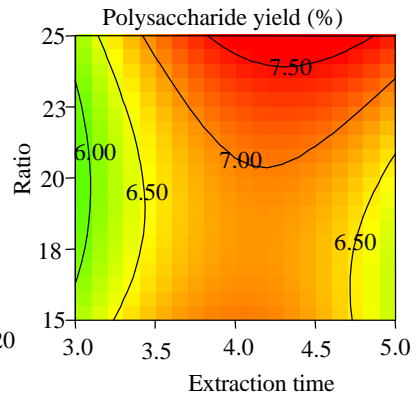

Fig. 2: Response surface plot and contour of ratio of water to raw matierial and extraction time and their mutual interactions on the yield of polysaccharides
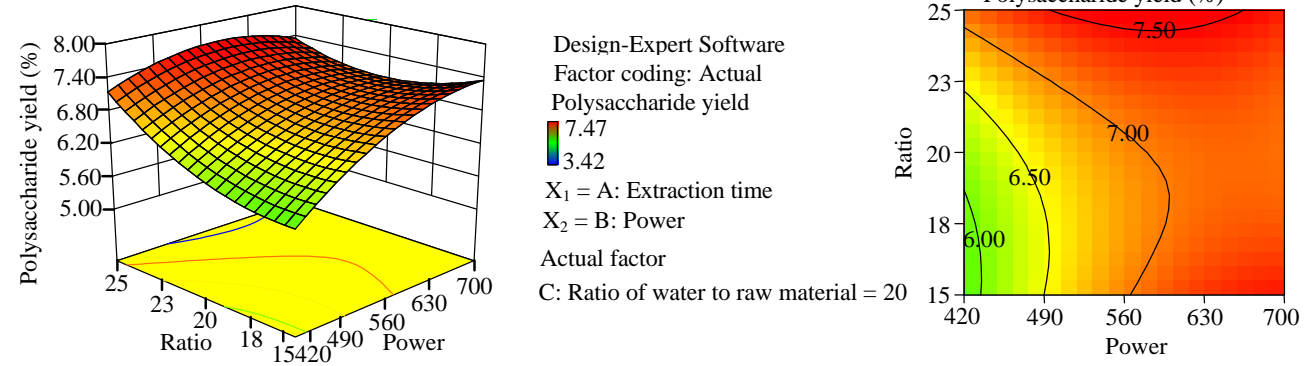

Fig. 3: Response surface plot and contour of ratio of water to raw material and microwave power and their mutual interactions on the yield of polysaccharides

\begin{tabular}{lcc} 
Table 5: Fit statistics for $y$ & \\
\hline Parameters & Master model & Predictive model \\
\hline Mean & 6.5400 & 6.5400 \\
$\mathrm{R}^{2}$ & 0.8808 & 0.8808 \\
Adj. $\mathrm{R}^{2}$ & 0.7276 & 0.7276 \\
RMSE & 0.5100 & 0.5100 \\
CV $(\%)$ & 7.8600 & 7.8600 \\
\hline
\end{tabular}

polysaccharides rapidly increased with increasing of microwave power from 420-700 W. According to Fig. 1-3, optimal extraction condition of MCP were extraction time $5 \mathrm{~min}$, microwave power $420 \mathrm{~W}$, ratio of water to raw material 25:1, respectively.

Among the three extraction parameters studied, the microwave power was the major factor affecting the extraction yield of MCP, followed by extraction time and ratio of water to raw material according to the regression coefficients significance of the quadratic polynomial model (Table 5) and gradient of slope in the 3-D response surface plot (Fig. 1-3).

The result was similar with previous investigation (Wang et al., 2010). During the MAE procedure, microwave energy is used to heat solvents in contact with samples and to partition analytes from sample matrix into the solvent. Microwave heating occurs in a targeted and selective manner with practically no heat being lost to the environment as the heating occurs in a closed system. This particular heating mechanism can significantly reduce the extraction time when compared to conventional heating (Mandal et al., 2007).
Table 6: Predicted and experimental values of the responses at optimum and modified conditions

\begin{tabular}{lcccl}
\hline Conditions & $\begin{array}{l}\text { Extraction } \\
\text { time (min) }\end{array}$ & $\begin{array}{l}\text { Microwave } \\
\text { power }(\mathrm{W})\end{array}$ & $\begin{array}{c}\text { Ratio of water } \\
\text { to raw material }\end{array}$ & Yield (\%) \\
\hline Optimum & 5 & 420 & 25 & 8.11 \\
Experimental & 5 & 420 & 25 & $7.83 \pm 0.19$ \\
\hline
\end{tabular}

Validation of the models: In order to validate the adequacy of the model equations (Eq. 2), a verification experiment was carried out using the recommended optimum conditions. The maximum predicted yield and experimental yield of Catathelasma ventricosum polysaccharides were shown in Table 6. Under the conditions, the experimental yield of polysaccharide was $7.83 \pm 0.19 \%(n=3)$ which was close to the predicted value. The good correlation between these results confirmed that the model was adequate for reflecting the expected optimization.

\section{CONCLUSION}

In this study, RSM was successfully used to determine the microwave-assisted extraction conditions for MCP. The optimal extraction conditions for the polysaccharides were as follows: extraction time $5 \mathrm{~min}$, microwave power $420 \mathrm{~W}$, ratio of water to raw material $25: 1$. Under these conditions, the experimental yield of polysaccharides was $7.83 \pm 0.19 \%$ which was close with the predicted yield value (Table 6). 
Agric. J., 7 (4): 255-259, 2012

\section{ACKNOWLEDGEMENTS}

This research was supported by the Department of Science and Technology of Sichuan Province of China (No. 09ZA083) and the Program for Changjiang Scholars and Innovative Research Team in University (No. IRT0848).

\section{REFERENCES}

Bagherian, H., F.Z. Ashtiani, A. Fouladitajar and M. Mohtashamy, 2011. Comparisons between conventional, microwave-and ultrasound-assisted methods for extraction of pectin from grapefruit. Chem. Eng. Process. Process. Intensi?cation, 50: $1237-1243$.

Bin, C., 2010. Optimization of extraction of Tremella fuciformis polysaccharides and its antioxidant and antitumour activities in vitro. Carbohydr. Polym., 81: $420-424$.

Buldini, P.L., R. Ricci and J.L. Sharma, 2002. Recent applications of sample preparation techniques in food analysis. J. Chromatogr. A, 975: 47-70.

Chen, Y., X. Gu, S. Huang, J. Li, X. Wang and J. Tang, 2010. Optimization of ultrasonic/microwave assisted extraction (UMAE) of polysaccharides from Inonotus obliquus and evaluation of its anti-tumor activities. Int. J. Biol. Macromol., 46: 429-435.

Diniz, R.O., L.K. Garla, J.M. Schneedorf and J.C. Carvalho, 2003. Study of anti-in?ammatory activity of Tibetan mushroom, a symbiotic culture of bacteria and fungi encapsulated into a polysaccharide matrix. Pharmacol. Res., 47: 49-52.

DuBois, M., K.A. Gilles, J.K. Hamilton, P.A. Rebers and F. Smith, 1956. Colorimetric method for determination of sugars and related substances. Anal. Chem., 28: $350-356$.

Liu, J., S. Miao, X. Wen and Y. Sun, 2009. Optimization of polysaccharides (ABP) extraction from the fruiting bodies of Agaricus blazei Murill using response surface methodology (RSM). Carbohydr. Polym., 78: 704-709.
Mandal, V., Y. Mohan and S. Hemalatha, 2007. Microwave assisted extraction: An innovative and promising extraction tool for medicinal plant research. Pharmacogn. Rev., 1: 7-18.

Pan, Y., Y. Hao, T. Chu, C. Li, Z. Zhang and Y. Zhou, 2010. Ultrasonic-assisted extraction, chemical characterization of polysaccharides from Yunzhi mushroom and its effect on osteoblast cells. Carbohydrate Polymers, 80: 922-926.

Sun, Y, S. Wang, T. Li, X. Li, L. Jiao and L. Zhang, 2008. PuriWcation, structure and immunobiological activity of a new water-soluble polysaccharide from the mycelium of Polyporus albicans (Imaz.) Teng. Bioresour. Technol., 99: 900-904.

Sun, Y., J. Liu and J.F. Kennedy, 2010. Application of response surface methodology for optimization of polysaccharides production parameters from the roots of Codonopsis pilosula by a central composite design. Carbohydrate Polymers, 80: 949-953.

Wang, J., J. Zhang, X. Wang, B. Zhao, Y. Wu and J. Yao, 2009. A comparison study on microwave-assisted extraction of Artemisia sphaerocephala polysaccharides with conventional method: Molecule structure and antioxidant activities evaluation. Int. J. Biol. Macromol., 45: 483-492.

Wang, J., J. Zhang, B. Zhao, X. Wang, Y. Wu and J. Yao, 2010. A comparison study on microwave-assisted extraction of Potentilla anserina L. polysaccharides with conventional method: Molecule weight and antioxidant activities evaluation. Carbohydrate Polymers, 80: 84-93.

Ye, C.L. and C.J. Jiang, 2011. Optimization of extraction process of crude polysaccharides from Plantago Asiatica L. by response surface methodology. Carbohydr. Polym., 84: 495-502.

Zhang, J., S. Jia, Y. Liu, S. Wu and J. Ran, 2011. Optimization of enzyme-assisted extraction of the Lycium barbarum polysaccharides using response surface methodology. Carbohydr. Polym., 86: 1089-1092. 